\title{
Produtividade de três cultivares de cana-de-açúcar sob manejos de sequeiro e irrigado por gotejamento
}

\author{
G lauber J. de C. G ava ${ }^{1}$, Marcelo de A. Silva ${ }^{1}$, Rodrigo C. da Silva ${ }^{2}$, Elisangela M. Jeronimo ${ }^{1}$, \\ Juliana C. S. Cruz ${ }^{1} \& O$ riel T. Kölln ${ }^{3}$
}

\begin{abstract}
RESUMO
O objetivo neste trabalho foi estudar o efeito da tecnologia de irrigação por gotejamento, em cultivares de cana-de-açúcar, em dois ciclos de produção (cana-planta e cana-soca). 0 delineamento experimental utilizado foi o de blocos ao acaso, com quatro repetições, constituídos pela combinação de três cultivares de cana-de-açúcar: RB867515; RB855536 e SP80-3280, e dois manejos da cultura: sistema de irrigação por gotejamento subterrâneo e sistema de sequeiro, totalizando seis tratamentos. 0 primeiro ciclo teve duração de 336 dias, ocorrendo precipitação de $1.480 \mathrm{~mm}$. 0 volume de água disponibilizado pelo sistema de irrigação por gotejamento foi de $400 \mathrm{~mm}$, totalizando $1.880 \mathrm{~mm}$. O segundo ciclo teve duração de 365 dias, cujo volume de água por meio de precipitação foi de 1.394 mm; somados aos 320 $\mathrm{mm}$ fornecidos pelo sistema de irrigação, totalizaram $1.714 \mathrm{~mm}$. O correu interação entre manejo e cultivar para as variáveis: produtividade de colmos (TCH) e produtividade de açúcar (TPH) em que a maior diferença foi observada para a cultivar SP80-3280. As cultivares apresentaram respostas diferenciadas na eficiência de utilização da água. No manejo irrigado por gotejamento houve elevação de $24 \%$ na produtividade de colmos e de $23 \%$ na produtividade de açúcar, em relação ao manejo de sequeiro.
\end{abstract}

Palavras-chave: Saccharum spp., disponibilidade hídrica, irrigação, produção

\section{Productivity of three sugarcane cultivars under dry and drip irrigated management}

\begin{abstract}
This study aimed to evaluate the effect of drip irrigation technology in different sugarcane varieties in two crop cycles (plant cane and ratoon). The experimental design was in completly randomized blocks, in split-plot with four replications, constituted by three sugarcane genotypes: RB867515; RB855536 and SP80-3280 and tw o crop management: drip irrigation system and rainfed system, totalizing six treatments. The first cycle lasted for 336 days, with rainfall of 1,480 $\mathrm{mm}$. The volume of water provided by the system of drip irrigation was $400 \mathrm{~mm}$, totaling $1,880 \mathrm{~mm}$. The second cycle lasted for 365 days, the volume of water through rainfall was $1,394 \mathrm{~mm}$, added to $320 \mathrm{~mm}$ provided by the system of irrigation, totaled $1,714 \mathrm{~mm}$. Interaction between management and cultivars was found significant for the variables: productivity of stalks (TCH) and sugar yield (TPH), in which the largest difference was observed for cultivar SP80-3280. There was significant response to drip irrigation, on average the increase of production of stalks and sugar was 24 and $23 \%$, respectively.
\end{abstract}

Key words: Saccharum spp., water availability, irrigation, yield

\footnotetext{
${ }^{1}$ Pesquisadores, APTA - Polo Centro O este, Rodovia Jaú, Bariri, SP, Km 304. CP 66, CEP 17201-970, Jaú, SP, Fone: (14) 3621 - 3439. E-mail: ggava@apta.sp.gov.br

2 D outorando, ESALQ/U SP, Av. Pádua Dias, 11. CEP 13418-900, Piracicaba, SP. Fone: (19) 3417-2100. E-mail: rcdsilva@esalq.usp.br

${ }^{3}$ M estrando, CEN A/USP, Av. Centenário, 303. CEP 13400-970, Piracicaba, SP, Fone: (19) 3429-4770. E-mail: orielkolln@cena.usp.br
} 


\section{INTRODUÇÃO}

A água é essencial para a produção vegetal, pois tanto a falta quanto o excesso prejudicam o crescimento e o desenvolvimento das plantas.

O déficit hídrico não é limitado apenas às regiões áridas e semiáridas do mundo uma vez que, mesmo em regiões consideradas climaticamente úmidas, a distribuição irregular das chuvas pode, em alguns períodos, limitar o crescimento (Ometto, 1980).

Silva et al. (2008) relataram que maior produtividade de canade-açúcar foi observada para a cultivar que manteve maiores atributos biométricos, tais como número massa e altura de colmos sob condições de estresse hídrico provocado por seca. Ainda a falta de água reduz a quantidade e a razão da fluorescência $(\mathrm{Fv} / \mathrm{Fm})$ da clorofila e o conteúdo relativo de água nas folhas, variáveis correlacionadas com produtividade (Silva et al., 2007). Assim, o manejo racional da água na cultura de cana-de-açúcar é fundamental para a maximização da produção (Dantas Neto et al., 2006).

A necessidade hídrica da cana-de-açúcar varia conforme os diferentes estádios fenológicos da cultura. Segundo Ometto (1980), dependendo do clima o volume de água de que a cultura necessita, varia de 1.500 e $2.500 \mathrm{~mm}$. Foligata (1974) verificou que, de acordo com as condições climáticas de Tucumán, na Argentina, a cana-de-açúcar careceu de 12,5 a 14,9 mm de água para produzir uma tonelada de colmos.

Nas áreas canavieiras do Brasil o total de precipitação anual varia de 1.100 a $1.500 \mathrm{~mm}$ nno $^{-1}$ (Alfonsi et al., 1987). Diante disso, Dantas Neto et al. (2006) e Farias et al. (2008) afirmaram que para a obtenção de produtividade elevada atingindo o potencial genético da cultura, o uso da tecnologia de irrigação é imprescindível.

A resposta de produtividade da cana-de-açúcar irrigada depende de um conjunto de fatores, dentre eles: da quantidade de água e de fertilizantes aplicados (Thorburn et al., 2003; Dantas Neto et al., 2006), do manejo de irrigação (Ramesh et al., 1994), da cultivar, idade de corte e do tipo de solo e do clima (Ramesh \& Mahadevaswamy, 2000; Smit \& Singels, 2006).

Portanto, o presente trabalho teve como objetivo estudar o efeito da tecnologia de irrigação por gotejamento, em diferentes cultivares de cana-de-açúcar, em dois ciclos de produção (cana-planta e cana-soca).

\section{Material e MÉTOdos}

O experimento foi conduzido na Unidade de Pesquisa e Desenvolvimento de Jaú, SP da Agência Paulista de Tecnologia dos Agronegócios, Polo Centro Oeste, localizado na latitude de $22^{\circ} 17^{\prime} \mathrm{S}$, e longitude $48^{\circ} 34^{\prime} \mathrm{W}$ e altitude média de $580 \mathrm{~m}$. O solo da área é Argissolo eutrófico, cujas características químicas analisadas na camada de $0-25 \mathrm{~cm}$, apresentaram: $\mathrm{pH}\left(\mathrm{CaCl}_{2}\right)$ 5,$2 ; \mathrm{P}$ (resina) $19,0 \mathrm{mg} \mathrm{dm}^{-3} ; \mathrm{K} 0,9 \mathrm{mmol}_{\mathrm{c}} \mathrm{dm}^{-3} ; \mathrm{Ca}_{2} 7,0 \mathrm{mmol}_{\mathrm{c}} \mathrm{dm}^{-3}$;

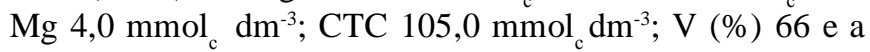
composição física de areia, silte e argila, foi $660,70,270 \mathrm{~g} \mathrm{~kg}^{-1}$, respectivamente. $\mathrm{O}$ delineamento experimental utilizado foi o de blocos ao acaso, com quatro repetições, constituídos pela combinação de três cultivares de cana-de-açúcar: RB867515 (C1); RB855536 (C2) e SP80-3280 (C3) e dois manejos da cultura: sistema de irrigação por gotejamento subterrâneo (I) e sistema de sequeiro (S), totalizando seis tratamentos.

Todos os tratamentos receberam uma dose de $180 \mathrm{~kg} \mathrm{ha}^{-1} \mathrm{de}$ $\mathrm{P}$ na forma de superfosfato simples aplicado no plantio, $220 \mathrm{~kg}$ $\mathrm{ha}^{-1} \mathrm{~K}_{2} \mathrm{O}$ na forma de cloreto de potássio e $150 \mathrm{~kg} \mathrm{ha}^{-1} \mathrm{~N}$ na forma de uréia. A aplicação de $\mathrm{N}$ e $\mathrm{K}$ nos tratamentos irrigados foi realizada por meio de fertirrigação, ao longo do desenvolvimento da cultura. Nos tratamentos não irrigados (sequeiro), esta aplicação foi parcelada em dois momentos, no plantio e aos 30 dias após.

Após a colheita da cana-planta, deu-se continuidade ao estudo, no primeiro ciclo de cana-soca. Assim, no ciclo da soqueira os tratamentos receberam $140 \mathrm{~kg} \mathrm{ha}^{-1} \mathrm{~N}$ (uréia), e 150 $\mathrm{kg} \mathrm{ha}^{-1} \mathrm{~K}_{2} \mathrm{O}$ (cloreto de potássio) aplicados por meio de fertirrigação, ao longo do desenvolvimento da cultura, no sistema irrigado. No manejo de sequeiro realizou-se a aplicação dos fertilizantes 40 dias após o corte da cana-planta com as mesmas doses da fertirigação.

As parcelas se constituíram de cinco linhas de cana de $30 \mathrm{~m}$ (área da parcela de $270 \mathrm{~m}^{2}$ ), sendo a área útil composta por três linhas centrais de cana, menos $2,5 \mathrm{~m}$ de comprimento de cada extremidade da parcela, totalizando $25 \mathrm{~m}$ (área útil da parcela de $135 \mathrm{~m}^{2}$ ). Em todos os tratamentos se utilizou o plantio em linha dupla (plantio em "W" ou "abacaxi"), com espaçamento de $1,80 \mathrm{~m}$ entre as linhas duplas; nos tratamentos irrigados o tubo gotejador foi enterrado a $20 \mathrm{~cm}$ de profundidade da superfície do solo, no meio da linha dupla, conforme a Figura 1. O tubo gotejador utilizado foi o DRIPNET PC 22135 FL vazão de 1.0 $\mathrm{L} \mathrm{h}^{-1}$ possuindo gotejadores a cada $0,5 \mathrm{~m}$.

Contabilizou-se o suprimento de água ao solo pelo somatório do volume de chuva (P) e de irrigação(I). A demanda atmosférica foi calculada pela evapotranspiração da cana-de-açúcar $(\mathrm{ETo} \times \mathrm{Kc}$ ), com um nível máximo de armazenamento ou capacidade de água disponível (CAD) de $100 \mathrm{~mm}$. Com esses dados se elaboraram estimativas de balaço hídrico decendial e se calcularam as deficiências hídricas (DEF), nos anos agrícolas

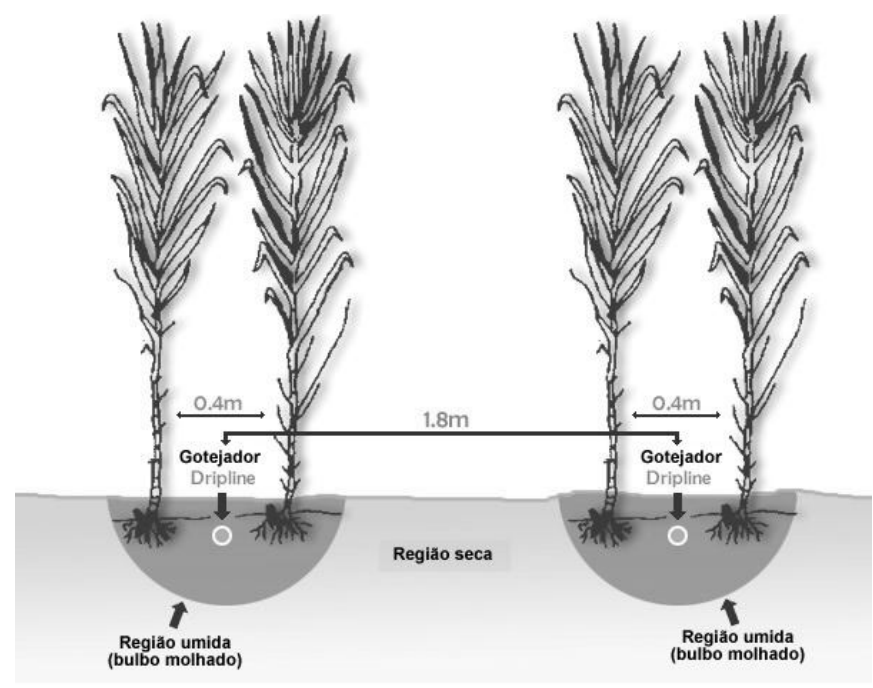

Figura 1. Instal ação dos tubos gotejadores nos tratamentos irrigados 
de 2006-2007 e 2007-2008, empregando-se o método de PenmanMonteith, cujos resultados estão apresentados na Figura 2.

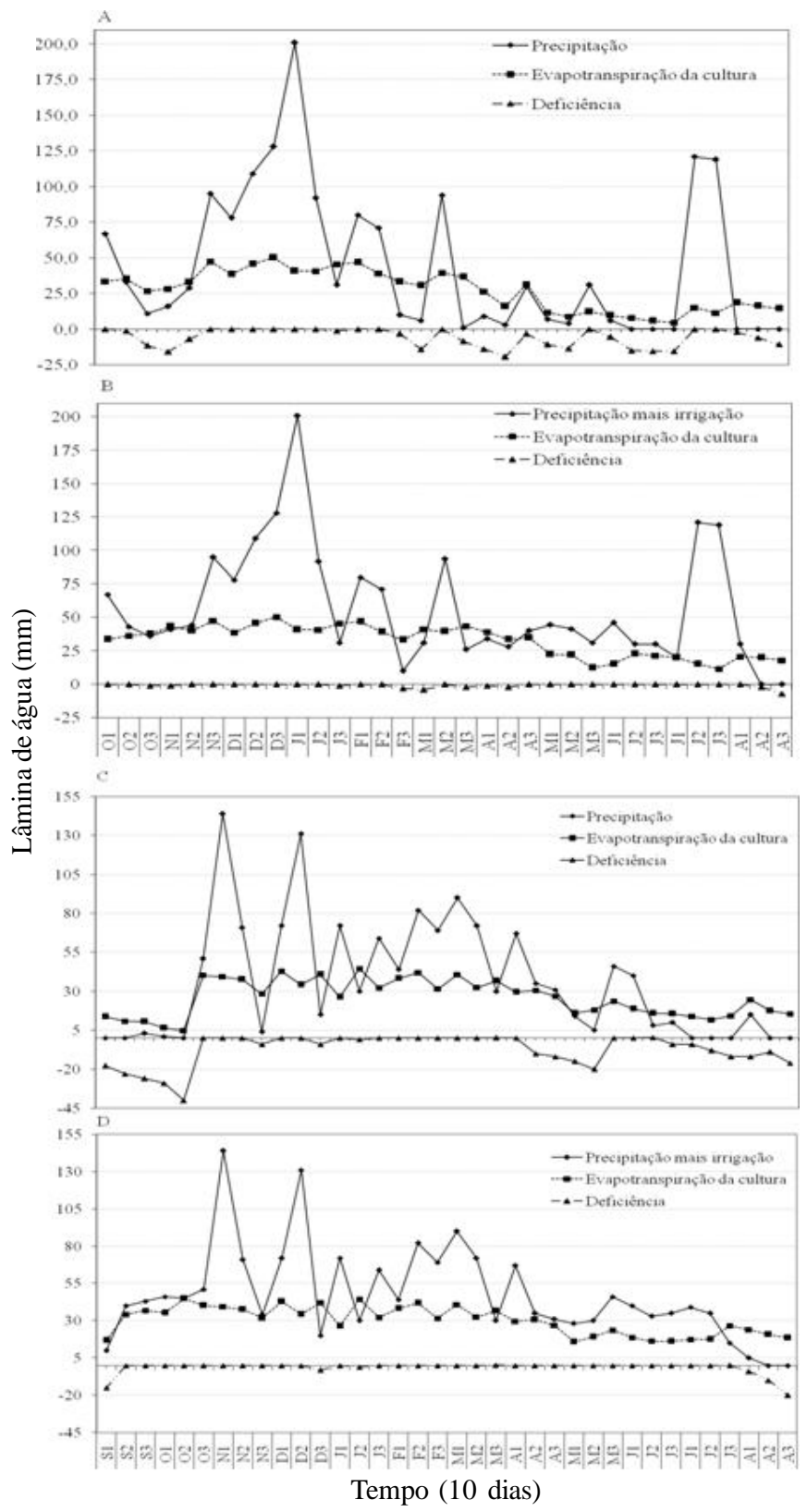

Figura 2. Balanço hídrico da cana-de-açúcar de sequeiro ( $A$ e $C$ ) e irrigado ( $B$ e $D$ ), nos ciclos de crescimento: 2006-2007 (A e B) e 2007-2008 (C e D)

Os resultados obtidos nos balanços hídricos (Figuras 2) apresentam a interação entre o clima e o desenvolvimento vegetal, nas duas safras estudadas (2006/2007 e 2007/2008), uma vez que o balanço hídrico fornece as estimativas da evapotranspiração da cultura $($ ETo $\times$ Kc) e deficiência hídrica (DEF).

O primeiro ciclo (cana-planta) teve duração de 336 dias, sendo que o volume de precipitação total foi de $1.480 \mathrm{~mm}$; a evapotranspiração no manejo de sequeiro foi de $902 \mathrm{~mm}$, apresentando déficit hídrico de 193 mm (Figura 2A). Neste mesmo período para o manejo irrigado, a precipitação somada ao volume de água disponibilizado pelo sistema de irrigação totalizou $1.880 \mathrm{~mm}$, com evapotranspiração de $1.070 \mathrm{~mm}$, apresentando um déficit de apenas $24 \mathrm{~mm}$ (Figura 2B).

O segundo ciclo de cultivo (2007/2008) teve duração de 365 dias, com ocorrência de precipitação total de $1.397 \mathrm{~mm}$ e evapotranspiração no manejo sequeiro de $927 \mathrm{~mm}$, com um déficit hídrico de $194 \mathrm{~mm}$ (Figura 2C). No manejo irrigado o volume de precipitação e o de irrigação somaram $1.714 \mathrm{~mm}$, com evapotranspiração de 1.083 mm e um déficit hídrico de 38 mm (Figura 2D).

Antes da colheita final foram amostrados 10 colmos em cada parcela para a realização de análises tecnológicas e obtenção dos valores de Pol\% de cana, Fibra \% de cana, conforme CONSECANA (2003).

A produtividade de colmos expressa em tonelada de colmos por hectare (TCH), foi obtida por meio da relação proporcional com a área de cada parcela. A tonelada de Pol por hectare (TPH) foi obtida pelo produto entre a produtividade de colmos (TCH) e do Pol\% da cana (PCC). Obteve-se a eficiência na utilização da água em relação à produtividade de colmos (EUA-TCH) através da quantidade de água em ( $\mathrm{mm})$ dividida pela produtividade de colmos em $\mathrm{t} \mathrm{ha}^{-1}$.

Os resultados foram submetidos a análise de variância utilizando-se o teste $\mathrm{F}$ a $5 \%$ e, para as causas de variações significativas, aplicou-se o teste de significância de Tukey (p $<0,05)$.

\section{RESULTADOS E DISCUSSÃO}

Houve efeito significativo da interação entre manejo (M) e cultivar (C), para as variáveis: produtividade de colmos $\left(\mathrm{F}=13,9^{*}\right)$, produtividade de açúcar $\left(\mathrm{F}=5,1^{*}\right)$ e eficiência de utilização de água em relação à produtividade de colmos $\left(\mathrm{F}=14,7^{*}\right)$, no ciclo da cana-planta e produtividade de colmos $\left(\mathrm{F}=10,2^{*}\right)$, e eficiência de utilização de água em relação à produtividade de colmos $\left(\mathrm{F}=10,7^{*}\right)$ na soma dos dois ciclos, não demonstrando efeito significativo para o ano de 2008, como demonstrado na Tabela 1. Esses resultados indicam uma relação direta entre a quantidade de água aplicada por meio da irrigação por gotejamento (manejo) nos diferentes genótipos de canade-açúcar estudados (cultivar).

A média de produtividade de colmos $(\mathrm{TCH})$ dos três genótipos estudados foi de $132,2 \mathrm{t} \mathrm{ha}^{-1}$ para o manejo irrigado por gotejamento e de $106,5 \mathrm{t} \mathrm{ha}^{-1}$ para o manejo de sequeiro, no primeiro ciclo de cultivo (2006/2007); no segundo ciclo (cana-soca), a média de TCH foi de 126,2 e 90,8 t ha-1, para os manejos irrigado e de sequeiro, respectivamente. De acordo com Doorembos \& Kassam (1994), o rendimento de cana-deaçúcar produzida em condições de sequeiro nos trópicos úmidos, varia entre 70 a 100 t ha $^{-1}$ e, nos trópicos e subtrópicos secos, com irrigação, entre 100 e $150 \mathrm{t} \mathrm{ha}^{-1}$, resultados que podem ser considerados satisfatórios. Neste experimento, a irrigação por gotejamento elevou a produtividade média em $20 \%$ no ciclo da cana-planta e de $28 \%$ no segundo ciclo (canasoca). Dalri (2004) obteve, em três ciclos de cultivo (canaplanta, $1^{\mathrm{a}}$ e $2^{\mathrm{a}}$ soca), cultivar RB72454, na região de Botucatu, $\mathrm{SP}$, uma produtividade média de $202 \mathrm{t} \mathrm{ha}^{-1}$ para cana irrigada por gotejamento e uma produtividade média de $145 \mathrm{t} \mathrm{ha}^{-1}$ para cana não irrigada. 
Tabela 1. Produtividade de colmos (TCH), produtividade de açúcar (TPH), e eficiência de utilização de água em relação à produtividade de colmos (EU A-TCH) de três cultivares de cana-de-açúcar, em manejo irrigado por gotejamento e no sequeiro

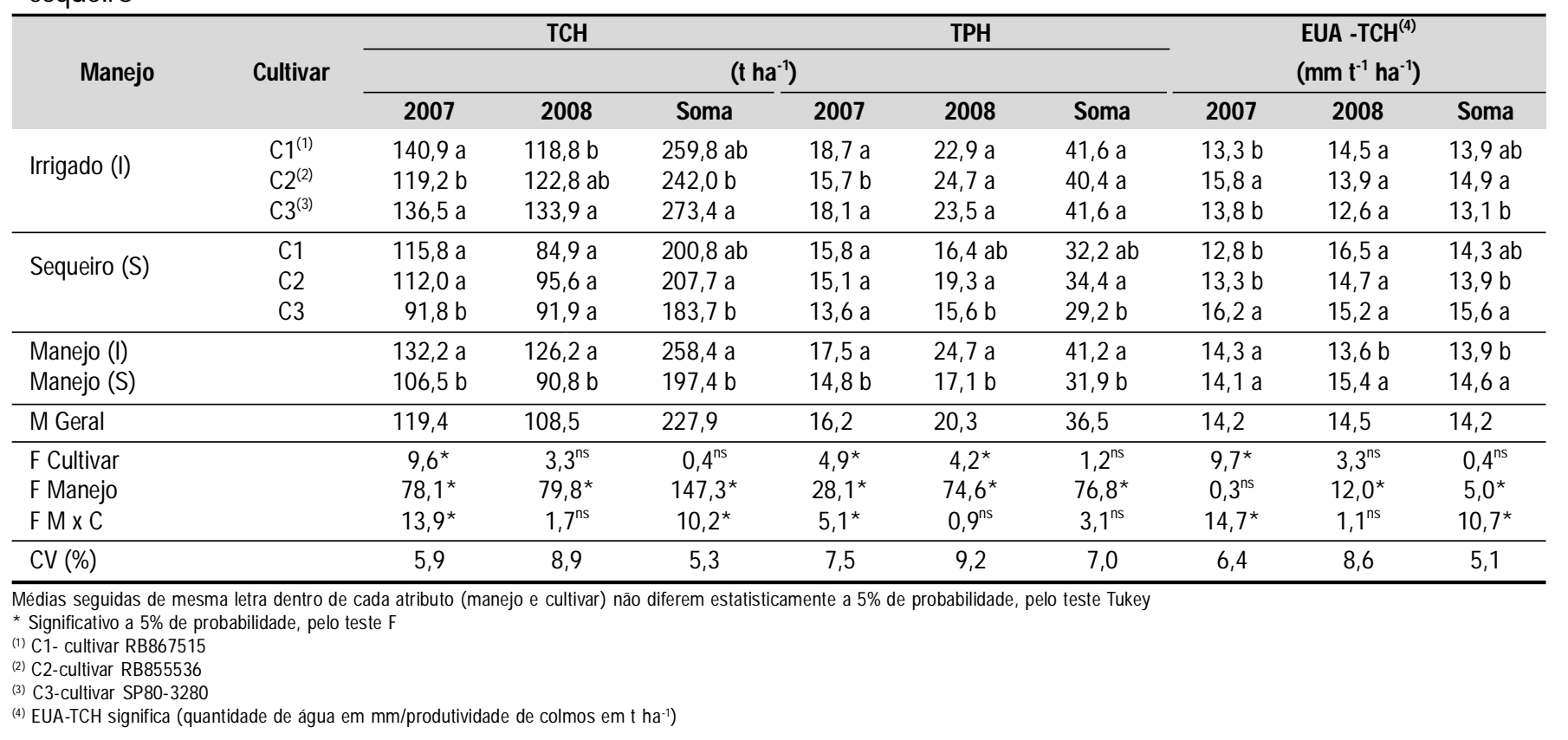

A produtividade de açúcar também foi acrescida em função da irrigação. A média de TPH dos três genótipos no manejo irrigado foi de 17,5 e 23,6 tha-1 de açúcar para os primeiro e segundo ciclos, respectivamente. A média dos três genótipos no manejo de sequeiro foi de 14,8 e 17,8 $\mathrm{tha}^{-1}$ para os primeiro e segundo ciclos, respectivamente (Tabela 1). Para os somatórios de produtividade de colmos e produtividade de açúcar observa-se um incremento na ordem de 24 e $23 \%$, respectivamente para os manejos irrigados por gotejamento e de sequeiro.

Pode-se inferir que o aumento da produtividade de colmos (TCH), no manejo de cana irrigada por gotejamento $(1.797 \mathrm{~mm})$ em comparação com o manejo de cana de sequeiro $(1.437 \mathrm{~mm})$, foi causado pela diminuição do estresse hídrico tendo em vista que a ocorrência desse tipo de estresse provoca, segundo alguns autores, redução de trocas gasosas (Farquhar et al., 1989; Inman-Bamber \& Smith, 2005); diminuição da área foliar e, consequentemente, do índice de área foliar (Inman-Bamber \& Smith, 2005; Smit \& Singels, 2006); redução no surgimento de novas folhas e aumento da abscisão foliar (Smit \& Singels, 2006) em virtude da elevação da concentração de ácido abscísico na planta (Ghannoum, 2009), que é associado à quantidade de luz absorvida e à fotossíntese total da cana-deaçúcar (Irvine, 1975), reduzindo a produção de fotassimilados (Farquhar et al., 1989; Lopes et al., 1988). Essas características fisiomorfológicas modificadas pelo estresse hídrico são consideradas de grande importância para se obter elevadas produtividades vegetais (Inman-Bamber \& Smith, 2005; Ghannoum, 2009).

Para as condições de campo deste experimento, a cana-deaçúcar necessitou, em média, de 14,2 mm de água para produzir uma tonelada de colmos (EUA-TCH), (Tabela 1); resultado semelhante a este foi verificado por Foligata (1974) e Singh et al. (2007).
Verifica-se, na Tabela 2, que no primeiro ciclo de cultivo (2006/2007) os valores de PCC foram inferiores aos do segundo ciclo (2007/2008), devido à chuva atípica no mês de julho de 2007 (Figura 2). A diminuição dos valores de PCC verificada no primeiro ciclo ocorreu, provavelmente, em vista das plantas terem sofrido menos estresse hídrico em relação ao segundo ciclo, de (2007/2008), concentrando menos açúcar.

$\mathrm{O}$ incremento da produtividade de açúcar (TPH) ocorreu devido ao maior acúmulo de biomassa pela cultura, no manejo

Tabela 2. Fibra \% de cana (F), pol \% de cana (PCC), de três cultivares de cana-de-açúcar, em manejo irrigado por gotejamento e no sequeiro, em dois ciclos de cultivo cana planta e cana soca

\begin{tabular}{|c|c|c|c|c|c|c|c|}
\hline \multirow{3}{*}{ Manejo } & \multirow{3}{*}{ Cultivar } & \multicolumn{3}{|c|}{$\mathbf{F}$} & \multicolumn{3}{|c|}{ PCC } \\
\hline & & \multicolumn{6}{|c|}{$(\%)$} \\
\hline & & 2007 & 2008 & Média & 2007 & 2008 & Média \\
\hline Irrigado (I) & $\begin{array}{l}\mathrm{C}^{(1)} \\
\mathrm{C2}^{(2)} \\
\mathrm{C}^{(3)}\end{array}$ & $\begin{array}{l}13,3 a \\
13,2 a \\
13,7 a\end{array}$ & $\begin{array}{l}13,3 \mathrm{a} \\
12,6 \mathrm{a} \\
12,4 \mathrm{a}\end{array}$ & $\begin{array}{l}13,3 a \\
12,9 a \\
13,1 \text { a }\end{array}$ & $\begin{array}{l}13,3 a \\
13,2 a \\
13,3 a\end{array}$ & $\begin{array}{l}15,9 a \\
16,8 \text { a } \\
17,1 a\end{array}$ & $\begin{array}{l}14,6 a \\
15,0 a \\
15,2 a\end{array}$ \\
\hline $\begin{array}{l}\text { Sequeiro } \\
\text { (S) }\end{array}$ & $\begin{array}{l}\mathrm{C} 1 \\
\mathrm{C} 2 \\
\mathrm{C} 3\end{array}$ & $\begin{array}{l}13,3 \mathrm{~b} \\
13,2 \mathrm{~b} \\
14,3 \mathrm{a}\end{array}$ & $\begin{array}{l}13,3 a \\
12,3 a \\
12,9 a\end{array}$ & $\begin{array}{l}13,3 \mathrm{ab} \\
12,7 \mathrm{~b} \\
13,6 \mathrm{a}\end{array}$ & $\begin{array}{l}13,6 \mathrm{~b} \\
13,5 \mathrm{~b} \\
14,8 \mathrm{a}\end{array}$ & $\begin{array}{l}15,9 a \\
16,9 a \\
16,9 a\end{array}$ & $\begin{array}{l}14,8 \mathrm{~b} \\
15,2 \mathrm{ab} \\
15,9 \mathrm{a}\end{array}$ \\
\hline $\begin{array}{l}\text { Manejo (I) } \\
\text { Manejo (S) }\end{array}$ & & $\begin{array}{l}13,4 \mathrm{a} \\
13,6 \mathrm{a}\end{array}$ & $\begin{array}{l}12,7 \mathrm{a} \\
12,8 \mathrm{a}\end{array}$ & $\begin{array}{l}13,1 \mathrm{a} \\
13,2 \mathrm{a}\end{array}$ & $\begin{array}{l}13,2 \mathrm{~b} \\
13,9 \mathrm{a}\end{array}$ & $\begin{array}{l}16,6 \mathrm{a} \\
16,6 \mathrm{a}\end{array}$ & $\begin{array}{l}14,9 \mathrm{a} \\
15,3 \mathrm{a}\end{array}$ \\
\hline Média geral & & 13,5 & 12,8 & 13,1 & 13,6 & 16,6 & 15,1 \\
\hline $\begin{array}{l}\text { F Cultivar } \\
\text { F Manejo } \\
\mathrm{FM} \times \mathrm{C}\end{array}$ & & $\begin{array}{l}7,4^{*} \\
0,9^{\text {ns }} \\
1,2^{\text {ns }} \\
\end{array}$ & $\begin{array}{c}2,9^{\text {ns }} \\
0,03^{\text {ns }} \\
0,5^{\text {ns }} \\
\end{array}$ & $\begin{array}{l}3,3^{\text {ns }} \\
0,4^{\text {ns }} \\
1,3^{\text {ns }}\end{array}$ & $\begin{array}{c}4,1^{*} \\
10,4^{*} \\
3,12^{\text {ns }} \\
\end{array}$ & $\begin{array}{c}4,0^{*} \\
0,0001^{*} \\
0,07^{\text {ns }} \\
\end{array}$ & $\begin{array}{l}6,9^{*} \\
3,7^{\text {ns }} \\
0,7^{\text {ns }} \\
\end{array}$ \\
\hline CV (\%) & & 3,5 & 5,9 & 3,5 & 4,1 & 5,0 & 3,0 \\
\hline
\end{tabular}

Médias seguidas de mesma letra dentro de cada atributo (manejo e cultivar) não diferem estatisticamente a $5 \%$ de probabilidade, pelo teste Tukey

* Significativo a $5 \%$ de probabilidade, pelo teste $\mathrm{F}$

(1) C1 - cultivar RB867515

(2) C2 - cultivar RB855536

(3) C3 - cultivar SP80-3280 
Tabela 3. Interação manejo $(\mathrm{m})$ versus cultivar (c) das variáveis: produtividade de colmos (TCH); produtividade de açúcar (TPH ); eficiência de utilização de água em relação à produtividade de colmos (EU A-TCH), em manejo irrigado por gotejamento e no sequeiro, em dois ciclos de cultivo (cana-planta e cana-soca)

\begin{tabular}{|c|c|c|c|c|c|c|c|c|c|c|}
\hline \multirow{3}{*}{ Cultivar } & \multirow{3}{*}{ Manejo $^{(1)}$} & \multicolumn{3}{|c|}{$\mathrm{TCH}$} & \multicolumn{3}{|c|}{ TPH } & \multirow{2}{*}{\multicolumn{3}{|c|}{$\begin{array}{l}\text { EUA- TCH } \\
\left(\mathrm{mm} \mathrm{t}^{-1} \mathrm{ha}^{-1}\right)\end{array}$}} \\
\hline & & \multicolumn{6}{|c|}{$\left(t h^{-1}\right)$} & & & \\
\hline & & 2007 & 2008 & Soma & 2007 & 2008 & Soma & 2007 & 2008 & Soma \\
\hline RB867515 & Sequeiro & $115,8 \mathrm{~b}$ & $84,9 a$ & $200,8 \mathrm{~b}$ & $15,8 \mathrm{~b}$ & $16,4 \mathrm{~b}$ & $32,2 \mathrm{~b}$ & $12,8 \mathrm{a}$ & $16,5 b$ & $14,3 a$ \\
\hline RB855536 & Irrigado & 119,2 a & $122,8 \mathrm{a}$ & $242,0 a$ & $15,7 \mathrm{a}$ & $24,7 a$ & $40,4 a$ & $15,8 \mathrm{a}$ & $13,9 a$ & $14,9 \mathrm{a}$ \\
\hline RB855536 & Sequeiro & 112,1 a & $95,6 \mathrm{~b}$ & $207,8 \mathrm{~b}$ & $15,1 \mathrm{a}$ & $19,3 \mathrm{~b}$ & $34,4 b$ & $13,3 b$ & $14,7 \mathrm{a}$ & $13,9 a$ \\
\hline
\end{tabular}

Médias seguidas de mesma letra dentro de cada atributo não diferem estatisticamente a $5 \%$ de probabilidade, pelo teste Tukey

(1) Manejo irrigado $1.880 \mathrm{~mm}$, manejo de sequeiro $1.480 \mathrm{~mm}$ no ano de 2007, manejo irrigado $1.714 \mathrm{~mm}$ manejo sequeiro $1.394 \mathrm{~mm}$ ano de 2008 , totalizando $3.594 \mathrm{~mm}$ no manejo irrigado, e 2.874 $\mathrm{mm}$ no manejo sequeiro

(2) EUA-TCH = (quantidade de água em $\mathrm{mm} /$ produtividade de colmos em $\mathrm{t}$ ha-1)

irrigado, pois os teores de sacarose da cana (PCC), não tiveram incrementos significativos em razão da irrigação por gotejamento subterrâneo (Tabela 2).

Os valores de \% de fibra não sofreram acréscimos significativos em função do manejo de irrigação por gotejamento; não houve diferença significativa entre cultivares (C) e diferentes períodos.

Além das características fisiomorfológicas de produtividade primária, citadas anteriormente, o manejo de irrigação por gotejamento (fertirrigação), aumenta a eficiência de utilização dos fertilizantes em comparação com o manejo de sequeiro (Thorburn et al., 2003). Este fato contribuiu, possivelmente, para a elevação da produtividade de colmos (TCH) no manejo irrigado (Tabela 1).

Na Tabela 3 se apresenta o desdobramento da interação manejo $(M)$ versus cultivar $(C)$, verificando-se que a irrigação por gotejamento produziu diferentes respostas entre os genótipos estudados.

A cultivar SP80-3280 elevou sua produtividade de colmos (TCH) em 33\%, em ambos os períodos e produtividade de açúcar (TPH), em 25 e 33\% nos períodos de 2007 e 2008 , respectivamente. Para a RB867515 observa-se um aumento de 18 e $15 \%$, respectivamente, para produtividade de colmos e de açúcar, no período de 2007 , e de $28 \%$ de produtividade de colmos e açúcar para o período de 2008. A cultivar RB855536, embora menor, também apresentou diferenças significativas em relação aos atributos avaliados, com elevação na ordem de $6 \%$ para produtividade de colmos $(\mathrm{TCH})$ e de $4 \%$ para a produtividade de açúcar (TPH) em 2007, mas no período de 2008 elevou a produtividade de colmos (TCH) e de açúcar (TPH) em $22 \%$, Tabela 3.

Em estudos realizados por outros autores, constatou-se que genótipos de cana-de-açúcar respondem diferentemente ao aumento da disponibilidade hídrica (Ramesh \& Mahadevaswamy, 2000; Inman-Bamber \& Smith, 2005; Smit \& Singels, 2006; Silva et al., 2007). Segundo Saliendra et al. (1996) e Inman-Bamber \& Smith (2005) a eficiência de utilização de água de uma cultivar de cana-de-açúcar está relacionada a fatores fisiológicos, como: capacidade de discriminação do isótopo estável ${ }^{13} \mathrm{C}$ pela enzima ribulose-1,5-bifosfato; a condutância estomática e a partição de fotoassimilados, entre outros, e morfológicos, como: índice de área foliar; elongação dos colmos e partição de matéria seca.
A eficiência de utilização de água (EUA-TCH) da cultivar SP80-3280 foi diferente para os manejos, irrigado e de sequeiro (Tabela 3). Este resultado demonstra que a referida cultivar é possivelmente, mais sensível ao estresse hídrico, comparandose com as demais (RB867515 e RB855536). A diferença de eficiência de utilização de água em relação à produtividade de colmos, verificada nos genótipos estudados, se deve sem dúvida, à capacidade que cada genótipo possui de tolerar o estresse hídrico e, em seguida, rapidamente se regenerar (Ramesh \& Mahadevaswamy, 2000; Smit \& Singels, 2006).

\section{Conclusões}

1. Ocorreu interação entre manejo (irrigação por gotejamento e sequeiro) e os genótipos (diferentes cultivares de cana-deaçúcar).

2. Houve resposta significativa da aplicação do sistema de irrigação por gotejamento, com incremento médio de $24 \%$ na produção dos colmos e de $23 \%$ na produção de açúcar.

3. Os genótipos de cana-de-açúcar RB867515, RB855536 e SP80-3280, responderam diferentemente ao aumento da disponibilidade hídrica.

\section{Agradecimentos}

À NETAFIM, ao Grupo COSAN, Unidade de Barra Bonita, e à Associação dos Produtores de Cana-de-açúcar da Região de Jaú (ASSOCICANA - Jaú).

\section{LITERATURA CITADA}

Alfonsi, R. R.; Pedro Júnior, M. J.; Brunini, O.; Barbieri, V. Condições climáticas para a cana-de-açúcar. In: Paranhos, S. B. (ed.). Cana-de-açúcar: Cultivo e utilização. Campinas: Fundação Cargill, v.1, p.42-55. 1987.

CONSECANA - Conselho dos Produtores de Cana-de-açúcar, Açúcar e Álcool do Estado de São Paulo. Manual de instruções. 4.ed. Piracicaba: CONSECANA, 2003. 115p. 
Dalri, A. B. Avaliação da produtividade da cana-de-açúcar irrigada por gotejamento subsuperficial nos três primeiros ciclos. Botucatu: UNESP, 2004. 89p. Tese Doutorado

Dantas Neto, J.; Figueirêdo, J. L. C.; Farias, C. H. A. de; Azevedo, H. M. de; Azevedo, C. A. V. de. Resposta da canade-açúcar, primeira soca, a níveis de irrigação e adubação de cobertura. Revista Brasileira de Engenharia Agrícola e Ambiental, v.10, p.283-288, 2006.

Doorembos, J.; Kassam, A. H. Efeito da água no rendimento das culturas. Campina Grande: UFPB, 1994. 306p. Estudos FAO. Irrigação e Drenagem, 33

Farias, C. H. A. de; Fernandes, P. D.; Azevedo, H. M. de; Dantas Neto, J. Índices de crescimento da cana-de-açúcar irrigada e de sequeiro no Estado da Paraíba. Revista Brasileira de Engenharia Agrícola e Ambiental, v.12, p.356-362, 2008.

Farquhar, G. D.; Ehleringer, J. R.; Hubick, K. T. Carbon isotope discrimination and photosynthesis. Annual Review of Plant Physiology and Plant Molecular Biology, v.40, p.530-537 1989.

Foligata, F. A. Sugarcane irrigation in Tucumán. In: Internacional Society Sugarcane Technologists, 15, 1974, Buenos Aires. Proceedings... Buenos Aires: Heyne \& Gibson, 1974. p.665-667.

Ghannoum, O. $\mathrm{C}_{4}$ Photosynthesis and water stress. Annals of Botany, v.103, p.635-644, 2009.

Inman-Bamber, N. G.; Smith, D. M. Water relations in sugarcane and response to water deficits. Field Crops Research, v.92, p.185-202, 2005.

Irvine, J. E. Relations of photosynthetic rates and leaf and canopy characters to sugarcane yield. Crop Science, v.15, p.671-676, 1975.

Lopes, B. F.; Seter, T. L.; Mcdavid, C. R. Photosynthesis and water vapor exchange of pingeonpea leaves in response to water deficit and recover. Crop Science, v.28, p.141-145, 1988.
Ometto, J. C. Parâmetros meteorológicos e a cultura da cana-de-açúcar. Piracicaba: ESALQ, 1980. 17p.

Ramesh, P.; Kailasam, C.; Srinivasan, T. R. Performance of sugarcane (Saccharum officinarum $\mathrm{L}$ ) under surface drip, sub surface drip (biwall) and furrow methods of irrigation. Journal of Agronomy and Crop Science, v.172, p.237-241, 1994.

Ramesh, P.; Mahadevaswamy, M. Effect of formative phase drought on different classes of shoots, shoot mortality, cane attributes, yield and quality of four sugarcane cultivars. Journal of Agronomy and Crop Science, v.185, p.249-258, 2000.

Saliendra, N. Z.; Meinzer, F. C.; Perry, M.; Thom, M. Associations between partitioning of carboxylase activity and bundle sheath leakiness to $\mathrm{CO}_{2}$, carbon isotope discrimination, photosynthesis, and growth in sugarcane. Journal of Experimental Botany, v.47, p.907-914, 1996.

Silva, M. A.; Jifon, J. L.; Silva, J. A. G.; Sharma, V. Use of physiological parameters as fast tools to screen for drought tolerance in sugarcane. Brazilian Journal of Plant Physiology, v.19, p.193-201, 2007.

Silva, M. A.; Silva, J. A. G.; Enciso, J.; Sharma, V.; Jifon, J. Yield components as indicators of drought tolerance of sugarcane. Scientia Agricola, v.65, p.620-627, 2008.

Singh, P. N.; Shukla, S. K.; Bhatnagar, V. K. Optimizing soil moisture regime to increase water use efficiency of sugarcane (Saccharum spp. Hybrid complex) in subtropical India. Agricultural Water Management, v.90, p.95-100, 2007.

Smit, M. A.; Singels, A. The response of sugarcane canopy development to water stress. Field Crops Research, v.98, p.91-97, 2006.

Thorburn, P. J.; Dart, I. K.; Biggs, I. M.; Baillie, C. P.; Smith, M. A.; Keating, B. A. The fate of nitrogen applied to sugarcane by trickle irrigation. Irrigation Science, v.22, p.201-209, 2003. 